\title{
Listériose humaine : Expériences cliniques avec cette zoonose chez 12 patients en Estrie, Québec sur une période de 19 ans (1976 à 1995)
}

PierRe-JeAn MaZiade MD, J ANDré MarCoux MD

\begin{abstract}
P-J MAZIADE, JA MARCOUX. Listériose humaine : Expériences cliniques avec cette zoonose chez 12 patients en Estrie sur une période de 19 ans (1976 à 1995). Can J Infect Dis 1996;8(1):29-32. Une étude rétrospective a été faite dans le but de réviser l'épidémiologie, les facteurs de risque, les manifestations cliniques et l'évolution des cas d'infection à Listeria monocytogenes en Estrie, Québec de 1976-1995. Les patients ont été repérés à partir des cultures positives et du diagnostic donné par le Service des maladies infectieuses du Centre hospitalier universitaire de Sherbrooke, Sherbrooke, Québec. Un total de 12 patients ont fait partie de l'étude. La septicémie non reliée à la grossesse (4/12) et la méningite (6/12) ont été les deux présentations cliniques majeures. Il y avait 1 cas de listériose de grossesse et 1 cas de granulomatosis infanti septica. Dix patients avaient au moins une condition prédisposante. Un seul décès a été attribué à l'infection à L monocytogenes. Des séquelles neurologiques ont été observées chez la moitié des patients avec méningite tandis qu'aucune séquelle a été notée chez ceux avec septicémie. En conclusion, la listériose est une maladie de patients âgés et immunocompromis. La présentation clinique et l'évolution ne sont pas différentes de ce qui a déjà été rapporté dans d'autres régions industrialisées.
\end{abstract}

Human listeriosis: Clinical experiences with 12 patients in the Eastern Townships, Quebec, over a 19-year period (1976 to 1995)

To review the epidemiology, risk factors, clinical features and outcomes of Listeria monocytogenes infection in the Eastern Townships of Quebec, a retrospective study was completed over the period 1976 to 1995. Cases were identified from positive cultures and diagnosis given by the infectious diseases service of the Centre Hospitalier Universitaire de Sherbrooke, Sherbrooke, Quebec. A total of 12 cases were in the study. Septicemia not related to pregnancy (four of 12) and meningitis (six of 12) were the major clinical presentations. There was one case of listeriosis associated with pregnancy and one case of granulomatosis infanti septica. Ten patients had at least one predisposing underlying condition. Only one death was related to L monocytogenes infection. Neurological sequelae were observed in half of the cases of meningitis, whereas no sequelae were noted in cases of septicemia. In conclusion, listeriosis is predominantly a disease of elderly and immunosuppressed patients. The clinical presentation and outcome are not different from those reported in other industrialized countries.

Key Words: Listeria monocytogenes, Listeriosis

Service de Maladies infectieuses, Département de médecine, Centre Universitaire de Santé de l'Estrie (CUSE), Sherbrooke, Quebec Correspondence: Dr P-J Maziade, Service de Maladies infectieuses, Département de médecine, Centre Universitaire de Santé de l'Estrie (CUSE), Sherbrooke, Quebec J1H 5N4.Telephone 819-563-5555, ext 14321, fax 819-564-5378, e-mail Amarcoux@courrier.usherb.ca

Received for publication October 26, 1995. Accepted April 19, 1996 
$\mathrm{L}$ isteria monocytogenes est le seul pathogène humain parmi les sept espèces de ce genre. C'est un bâtonnet Gram positif, aérobique et ne formant pas de spores. Il est mobile à la température de la pièce et il est hémolytique. A la coloration de Gram il peut ressembler aux streptococces, spécialement le pneumocoque. De plus, si la décoloration est trop forte, il peut être confondu avec un petit bâtonnet Gram négatif comme l'Haemophilus sp. Il fût la première fois isolé d'un liquide céphalorachidien d'un jeune soldat en 1918 par Dumont et Cotoni. Cette souche conservée pendant 20 ans, sera finalement identifiée à l'Institut Pasteur comme Listeria monocytogenes en 1938.

$L$ monocytogenes est répandu dans la nature. En effet, on l'a isolé dans le sol, la poussière, la nourriture, l'eau, les légumes, les animaux et l'homme.

Aux Ètaits-Unis, l'incidence annuelle de listériose est de 0.7 par 100,000 habitants (1). Les porteurs asymptomatiques au niveau des selles ont été estimés entre 1 et $5 \%$ (2). Toutefois, lorsqu'il s'agit de contacts de patients symptomatiques, les taux d'excréteurs peuvent atteindre $26 \%$. La listériose se présente habituellement sous forme de bactériémie ou de méningite. Les femmes enceintes et les immunocompromis constituent des groupes particulièrement à risque. Pour mieux comprendre les aspects epidémiologiques et cliniques de la listériose humaine dans notre milieu, nous avons révisé tous les cas de cette infection notés au Centre hospitalier universitaire de Sherbrooke (CHUS), Sherbrooke, Québec, et dans ses hôpitaux affiliés sur une période de 19 ans, soit de 1976 à 1995.

\section{MATÉRIEL ET MÉTHODES}

Nous ont été étudiés rétrospectivement les dossiers de tous les patients avec une (ou des) culture(s) positive(s) pour $L$ monocytogenes de février 1976 à juin 1995. Les patients ont été repérés à partir des cultures positives et du diagnostic porté par le Service des maladies infectieuses du CHUS. Sept paramètres principaux ont été relevés et analysés, soient: données démographiques, symptômes, conditions prédisposantes, site(s) de cultures positives, données paracliniques pertinentes, traitement et évolution.

\section{RÉSULTATS}

Un total de 12 cas ont été repertoriés dans les 19 dernières années en Estrie. Il s'agit de 8 hommes et 4 femmes. La moyenne d'âge est de 52,2 ans (excluant le cas néonatal). Toutefois, 7 des patients étaient âgés de 59 ans et plus. Depuis 197,5 des cas sporadiques de listériose ont été coustatés. Cependant, il est à noter que le tiers de nos cas ont été diagnostiqués dans les deux dernières années (1994-1995). Il semble y avoir une prédilection des cas de listériose dans les 6 derniers mois de l'année (juin à décembre). En effet, 91,7\% (11/12) des cas se sont échelonnés entre juin et décembre. L'incidence annuelle depuis 1975 est de 0,8 cas/100,000 habitants/année.

Un total de 4 cas de septicémies non reliées à la grossesse, 4 cas de méningites bactériémiques, 2 cas de méningites non bactériémiques, 1 cas de listériose pendant la grossesse et 1 cas de listériose néonatale (granulomatosis infanti septica) ont été observés. Les cas de méningites ont nécessité en moyenne 31,3 jours d'hospitalisation tandis que les septicémies ont nécessité 18,2 jours en moyenne.

Dix des patients avaient au moins une condition prédisposante (Table 1). Deux patients n'avaient aucune condition prédisposante identifiée. Il est à noter que 7 des patients étaient immunocompromis (grossesse, prématurité, cancer, chimiothérapie, corticostéroïdes, neutropénie et greffe rénale).

Au niveau des symptômes pour les patients atteints de méningite, la fièvre était présente chez tous (6/6). De plus, 4 de ces patients avaient des céphalées et la moitié des troubles de l'état de conscience (somnolence, stupeur et coma). A l'examen physique, une fièvre fut objectivée chez la majorité des patients de même qu'une raideur de nuque. De plus 2 patients sur 6 avaient des réflexes cutanés plantaires en extension bilatéraux (Babinsky).

Pour ce qui est des symptômes des patients qui ont présenté une septicémie, la fièvre était présente chez tous (5/5). De plus, 3 patients sur 5 ont noté des frissons ainsi que de la diarrhée. Aussi, 2 patients sur 5 se sont plaints de symptômes respiratoires supérieurs. L'examen physique était habituellement sans particularité à l'exception de la fièvre. En ce qui concerne le prématuré de 29 semaines avec la granulomatosis infanti septica, l'apnée et la détresse respiratoire étaient les symptômes majeurs. A l'examen physique, un rash typique caractérisé par des macules ou des taches érythémateuses pseudo-purpuriques, parfois centrées par un point blanc et disséminées sur tout le corps, a été noté. Le liquide amniotique était clair.

$\mathrm{Au}$ niveau paraclinique, 8 des patients présentaient une leucocytose $\left(12000 / \mathrm{mm}^{3}\right.$ à $\left.19000 / \mathrm{mm}^{3}\right)$ avec une prédominance de polymorphonucléaires (PMN). L'analyse du liquide céphalorachidien chez les patients atteints de méningite a démontré un décompte cellulaire entre 180 à 4000 globules blancs $(\mathrm{GB}) / \mathrm{mm}^{3}$. Chez 4 sur 6 de ces patients le décompte était supérieur à $500 \mathrm{~GB} / \mathrm{mm}^{3}$. De plus, 5 patients sur 6 avaient une prédominance de PMN (60 à 90\%). Tous les patients $(5 / 5)$ avaient une glycorachie inférieure aux 2/3 de la glycémie. La protéinorachie variait de 0,5 à 4 fois la normale (4 patients).

Le(s) site(s) de cultures positives pour $L$ monocytogenes a (ont) été: hémocultures chez 6 patients, hémocultures et le liquide céphalorachidien (LCR) chez 4 patients et LCR chez 2 patients. Il est à noter que la coloration de Gram sur le LCR était négative chez la majorité de nos patients (5/6). Le $L$ monocytogenes a également été trouvé au niveau du vagin et du placenta de la patiente enceinte ainsi qu'au niveau de la gorge, de la narine, de l'oreille, de l'oeil et du tube endotrachéal de son bébé.

Il y a eu guérison sans séquelle chez 7 patients sur 12 (3/6 méningites et $4 / 5$ septicémies). Des séquelles ont été notées chez 3 des 6 patients avec méningite et chez le nouveau-né (Table 1). Un décès a été indirectement associé à l'infection chez un patient avec quasi noyade, sous ventilation mécanique et syndrome de détresse respiratoire de l'adulte (SDRA).

Les traitements donnés chez nos patients ont été de l'ampicilline avec un aminoside (5/12), de la pénicilline (4/12), de l'ampicilline seule (1/12) et de l'érythromycine avec tobramycine (1/12). Un des patients n'a pas reçu de traitement. 
TABLE 1

Données sur 12 patients avec infection à Listeria monocytogenes en Estrie, Québec (1976-1995)

\begin{tabular}{|c|c|c|c|c|c|c|}
\hline $\begin{array}{l}\text { Âge (ans)/ } \\
\text { sexe }\end{array}$ & Diagnostic (mois) & $\begin{array}{c}\text { Durée } \\
\mathbf{d}^{\prime} \text { hospitalisation } \\
\text { (jours) }\end{array}$ & $\begin{array}{c}\text { Site de culture } \\
\text { positive }\end{array}$ & $\begin{array}{c}\text { Conditions } \\
\text { prédisposantes }\end{array}$ & Traitement (jours) & Séquelles \\
\hline $45 / M$ & Méningite (2) & 14 & LCR, sang & Lait non pasteurisé & Pénicilline (30) & $\begin{array}{l}\text { Dysarthrie, } \\
\text { diplopie }\end{array}$ \\
\hline $21 / F$ & Septicémie (10) & 14 & $\begin{array}{c}\text { Sang, vagin, } \\
\text { placenta }\end{array}$ & $\begin{array}{l}\text { Grossesse, lait } \\
\text { chèvre }\end{array}$ & Pénicilline (14) & Aucune \\
\hline $\begin{array}{l}\text { 29(semaines) } \\
\text { /M }\end{array}$ & $\begin{array}{c}\text { Listériose } \\
\text { néonatale (10) }\end{array}$ & 92 & $\begin{array}{l}\text { Sang, narine, } \\
\text { oreille, oeil }\end{array}$ & $\begin{array}{l}\text { Listériose chez } \\
\text { mère }\end{array}$ & $\begin{array}{l}\text { Ampicilline+ } \\
\text { aminoside (30) }\end{array}$ & Prématuré \\
\hline $74 / F$ & Septicémie (10) & 0 & Sang & Animaux, ferme & Aucun & Aucune \\
\hline $59 / M$ & Méningite (5) & 44 & LCR, sang & Gliosarcome & $\begin{array}{c}\text { Pénicilline+ } \\
\text { aminoside (30) }\end{array}$ & $\begin{array}{c}\text { SIHAD, aphasie, } \\
\text { Apraxie }\end{array}$ \\
\hline $20 / M$ & Septicémie (11) & 27 & Sang & $\begin{array}{l}\text { Hodgkin, } \\
\text { neutropénie }\end{array}$ & Pénicilline (21) & Aucune \\
\hline $80 / \mathrm{M}$ & Méningite (7) & 29 & LCR & Cancer prostate & $\begin{array}{l}\text { Ampicilline ( } 21)+ \\
\text { aminoside (7) }\end{array}$ & Aucune \\
\hline $75 / M$ & Méningite (11) & 23 & LCR & Nil & Pénicilline (21) & Aucune \\
\hline $59 / F$ & Septicémie (7) & 19 & Sang & $\begin{array}{l}\text { Greffe rénale, } \\
\text { stéroide, } \\
\text { cyclosporine }\end{array}$ & Ampicilline (14) & Aucune \\
\hline $67 / M$ & Méningite (10) & 26 & LCR, sang & LLC, stéroides & $\begin{array}{l}\text { Ampicilline+ } \\
\text { aminoside (21) }\end{array}$ & $\begin{array}{c}\text { Dysgraphesthésie, } \\
\text { dystéréognosie } \\
\text { gauche }\end{array}$ \\
\hline $2 / \mathrm{M}$ & Septicémie (12) & 11 & Sang & Noyade & $\begin{array}{l}\text { Ampicilline+ } \\
\text { aminoside (4) }\end{array}$ & SDRA \\
\hline $72 / F$ & Méningite (6) & 14 & LCR, sang & Nil & $\begin{array}{l}\text { Ampicilline }+ \\
\text { aminoside (21) }\end{array}$ & Aucune \\
\hline
\end{tabular}

LCR Liquide céphalorachidien; LLC Leucémie lymphoïde chronique; SDRA Syndrome de détresse respiratoire de l'adulte; SIHAD Sécrétion inappropriée d'hormone anti-diurétique

\section{DISCUSSION}

Dans un premier temps, il est intéressant de noter que l'incidence annuelle de listériose en Estrie n'est pas tellement différente de celle estimée aux États-Unis $(0,7$ cas/100,000 habitants/année) (1). De plus, nous avons noté que l'incidence augmente avec l'âge, un fait rapporté dans les études rétrospectives de cas récentes (3). Également, nous avons remarqué que la majorité de nos cas survenait dans les 6 derniers mois de l'année, ce qui est en accord avec la littérature à ce sujet $(3,4)$. Ceci contraste avec la listériose animale qui survient le plus souvent en hiver.

Dans notre étude, nous avons noté que seulement 2 cas sur 12 étaient associés à la grossesse. Malgré notre petit nombre de patients, ceci est nettement inférieur à ce qui est rapporté ailleurs (30\% à $45 \%$ des cas) $(3,5,6)$. Toutefois, notre patiente enceinte avait une présentation clinique typique avec des symptômes grippaux et de la diarrhée, ayant débuté au 3ième trimestre, le tout s'étant compliqué d'un travail préterme.

$\mathrm{Au}$ moins un condition prédispoaute était présente chez $83 \%$ de nos patients. Deux études récentes, une anglaise et l'autre australienne, mentionnent essentiellement les mêmes pourcentages ( $84 \%$ et $86 \%$ respectivement) $(3,7)$. Les conditions sous-jacentes de nos patients n'étaient pas différentes de celles rapportées dans la littérature.
Selon un sondage mené aux États-Unis (8), il y aurait jusqu'à $20 \%$ des cas de listériose qui seraient reliés à la nourriture, incluant les viandes mal cuites ou crues, les produits laitiers et les légumes. Dans la présente étude, 3 de nos cas étaient possiblement reliés à la nourriture (lait non pasteurisé). Au Canada, $3 \%$ des fromages, $7 \%$ des viandes et $12,8 \%$ des poissons contiennent du L monocytogenes (9).

Une condition prédisposante non trouvée chez nos patients est le syndrome d'immunodéficience acquis (SIDA). En effet, l'incidence de listériose dans cette population est de 65 à 145 fois plus élevée que dans la population générale (10). Ceci peut s'expliquer par le fait qu'en Estrie la prévalence du SIDA est faible.

Pour ce qui est des septicémies, les symptômes et signes présentés étaient non spécifiques. Toutefois, on remarque que 3 patients sur 5 se sont plaints de diarrhées. Ceci pourrait suggérer que la septicémie serait à départ intestinal.

En ce qui a trait aux méningites, la fièvre, des céphalées, un sensorium altéré et une raideur de nuque étaient de règle. La coloration de Gram sur le LCR était le plus souvent négative (5 patients/6). La littérature rapporte les mêmes constatations (7). En revanche, la culture était le plus souvent positive. L'analyse du LCR chez nos patients montrait une prédominance de neutrophiles, un fait bien connu dans les méningites à Listeria. Les méningites étaient bactériémiques 
chez 4 de nos 6 patients. Il semble que les hémocultures soient souvent positives dans les méningites à $L$ monocytogenes selon une étude récente australienne (7). Il est possible que ces patients avec meningite bactériémique avaient un certain degré de cérébrite. En effet, dans les cérébrites, les hémocultures sont souvent positives (11). De plus, on remarque que 3 de ces 4 patients avaient des signes neurologiques focaux avant et après le traitement, ce qui est typique de la cérébrite.

$\mathrm{Au}$ sujet du traitement, la pénicilline ou l'ampicilline étaient les premiers choix. Dans les études animales, l'ajout d'un aminoside est synergique. La durée du traitement peut varier de 2 à 4 semaines. Un traitement de 2 semaines serait

\section{BIBUOGRAPHIE}

1. Wenger JD, Hightower AW, Facklan RR, et al. Bacterial meningitis in the United States, 1986: Report of a multistate surveillance study. J Infect Dis 1990;162:1316-23.

2. Schuchat A, Deaver K, Hayes PG, et al. Gastrointestinal carriage of Listeria monocytogenes in household contacts of patients with listeriosis. J Infect Dis 1993;167:1261-2.

3. Jones EM, McCulloch SY, Reeves DS, et al. A 10 years survey of the epidemiology and clinical aspects of listeriosis in a provincial English city. J Infect 1994;29:91-103.

4. Stamm AM, Dismukes WE, Simmons BP, et al. Listeriosis in renal transplant recipients: Report of an outbreak and review of 102 cases. Rev Infect Dis 1982;4:665-81.

5. McLauchlin J. Human listeriosis in Britain, 1967-85, a summary of 722 cases. 1. Listeriosis in the pregnancy and the newborn. Epidemiol Infect 1990;104:181-90.

6. McLauchlin J. Human listeriosis in Britain, 1967-85, a summary of 722 cases. 2 . Listeriosis in nonpregnant individuals, a changing pattern of infection and seasonal incidence. Epidemiol Infect 1990;104:191-201. probablement suffisant pour une septicémie mais un traitement de 3 à 4 semaines serait plus approprié pour une méningite. L'alternative pour les patients allergiques à la pénicilline serait le triméthoprim-sulfaméthoxazole (12).

La mortalité chez nos patients (1/12) est nettement inférieure à celle rapportée dans la littérature (20\% à 30\%) $(3,5,7,9)$. Ceci peut s'expliquer par le fait que nous avons qu'un petit nombre de patients.

En conclusion, la listériose est une zoonose d'incidence faible, qui donne habituellement une septicémie ou une méningite chez des patients qui sont le plus souvent immunocompromis et âgés. La pénicilline ou l'ampicilline avec un aminoside demeure le traitement de premier choix.

7. Paul ML, Dwyer DE, Chow C, et al. Listeriosis - a review of eighty-four cases. Med J Aust 1994;160:489-93.

8. Schwartz B, Ciesielski CA, Broome CV, et al. Association of sporadic listeriosis with consumption of uncooked hot dogs and undercooked chicken. Lancet 1988;ii:779-82.

9. Farber JM, Harwig J, Carter A. Prevention of foodborne listeriosis. Can J Infect Dis 1991;2:116-20.

10. Jurado RL, Farley MM, Pereira E, et al. Increased risk of meningitis and bacteriemia due to Listeria monocytogenes in patients with human immunodeficiency virus infection. Clin Infect Dis 1993;17:224-7.

11. Mandell GL, Bennett JE, Dolin R. Principles and Practice of Infectious Diseases, 4th edn. New York: Churchill Livingstone, 1995:1883.

12. Spitzer PG, Hammer AM, Karchmer AW. Treatment of Listeria monocytogenes infection with trimethoprim-sulfamethoxazole: Case report and review of literature. Rev Infect Dis 1986;8:427-30. 


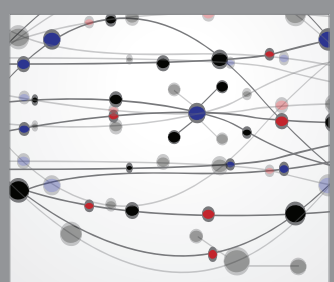

The Scientific World Journal
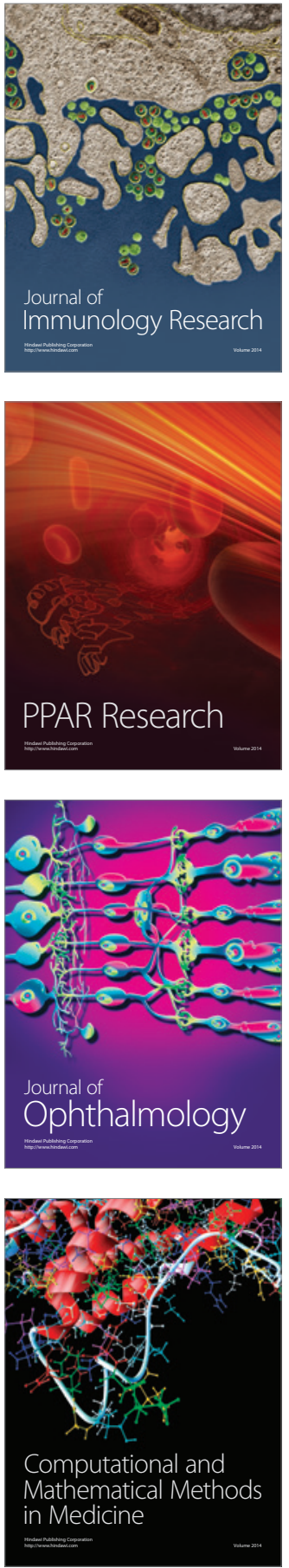

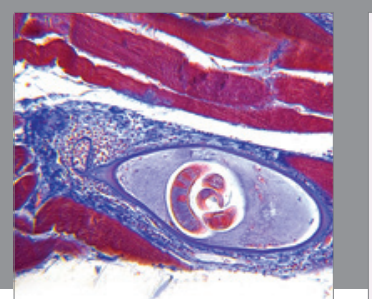

Gastroenterology Research and Practice

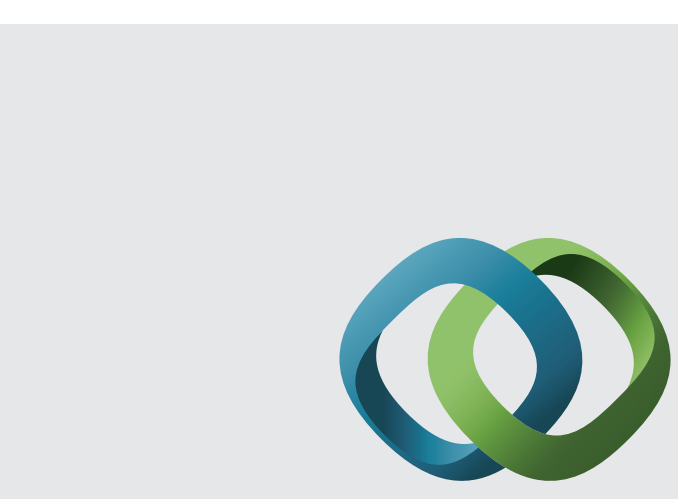

\section{Hindawi}

Submit your manuscripts at

http://www.hindawi.com
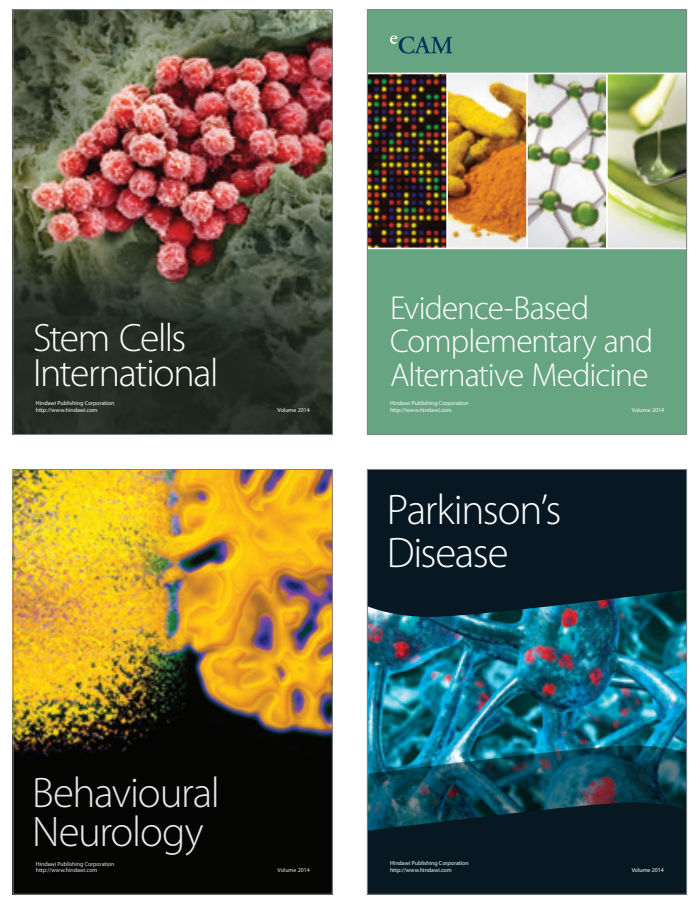
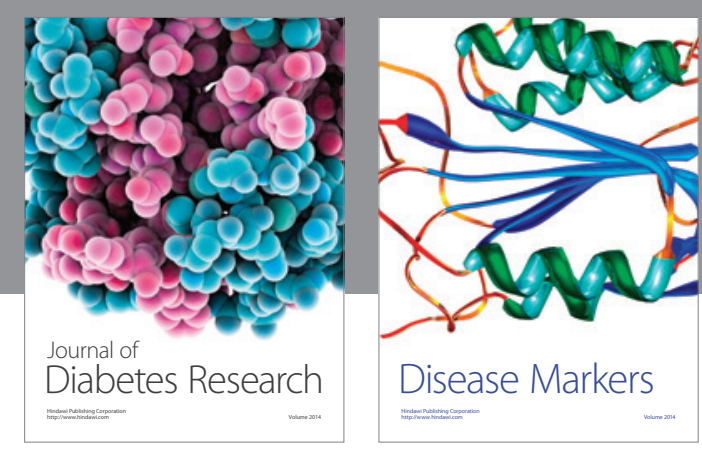

Disease Markers
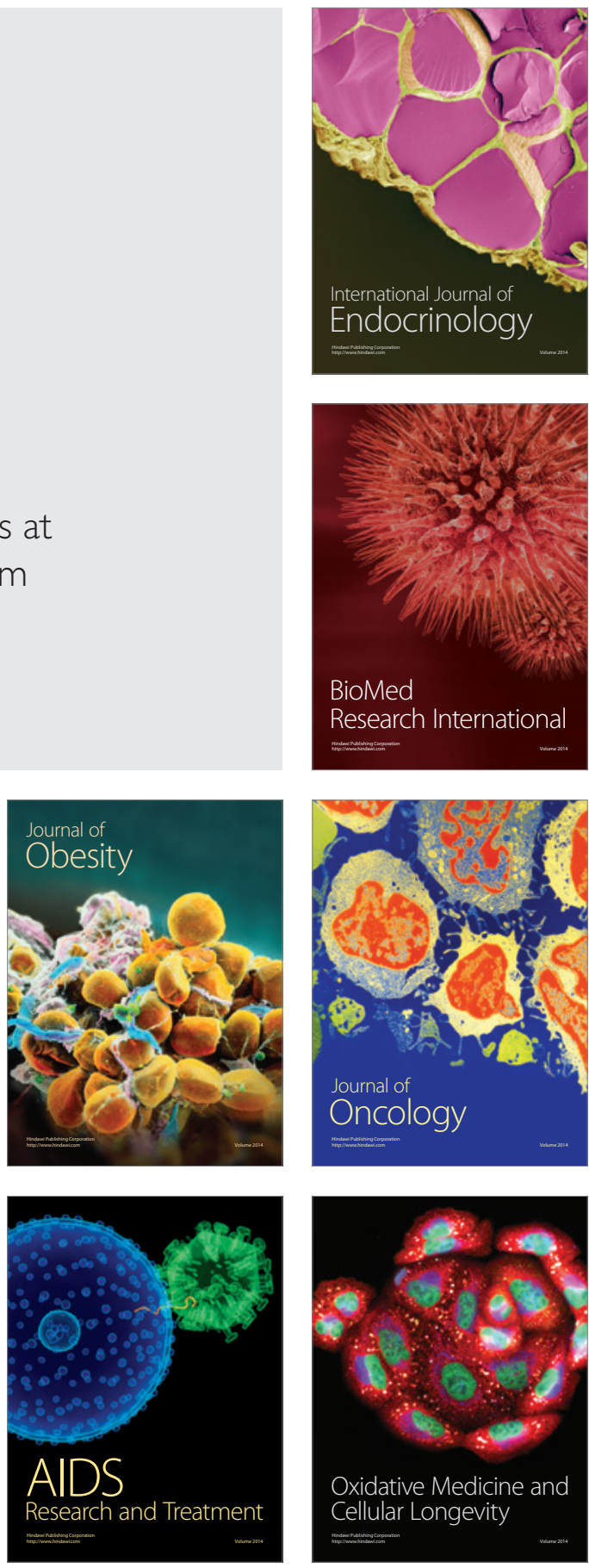